\title{
Nutrição mineral de mudas de pupunheira sob diferentes níveis de salinidade ${ }^{(1)}$
}

\author{
Antonio Rodrigues Fernandes ${ }^{(2)}$, Janice Guedes de Carvalho ${ }^{(3)}$, Nilton Curi(3), \\ José Eduardo Brasil Pereira Pinto ${ }^{(4)}$ e Paulo de Tácito Gontijo Guimarães ${ }^{(5)}$
}

\begin{abstract}
Resumo - O potencial produtivo das plantas pode ser afetado por desordens nutricionais, induzidas pelo estresse salino. O objetivo deste trabalho foi estudar a influência de diferentes níveis de salinidade na nutrição mineral de mudas de pupunheira (Bactris gasipaes H.B.K.), mediante o uso de solução nutritiva, em casa de vegetação. $\mathrm{O}$ experimento foi instalado em blocos ao acaso, com sete tratamentos e quatro repetições. Os tratamentos constaram das seguintes doses $\left(\mathrm{mmol} \mathrm{L}^{-1}\right): 0,0$ de $\mathrm{Na}$ e $0,5 \mathrm{de} \mathrm{Cl}$; 1,0 de $\mathrm{Na}$ e $0,5 \mathrm{de} \mathrm{Cl}$ (controle); e 5,0, 15,0, 30,0,60,0 e 120,0 de NaCl. Os teores dos macronutrientes variaram em razão da salinidade, e verificou-se que o $\mathrm{P}$ e $\mathrm{K}$ apresentaram seus teores reduzidos nas raízes, o $\mathrm{K}$ e o $\mathrm{Ca}$, nas folhas e o $\mathrm{S}$, nas diferentes partes, enquanto o $\mathrm{Na}$ e o $\mathrm{Cl}$ apresentaram teores incrementados nas diferentes partes da planta. As relações $\mathrm{Na} / \mathrm{K}, \mathrm{Na} / \mathrm{Ca}, \mathrm{Na} / \mathrm{Mg}, \mathrm{Cl} / \mathrm{N}, \mathrm{Cl} / \mathrm{P} \mathrm{e} \mathrm{Cl} / \mathrm{S}$ aumentaram, causando um desbalanço nutricional na planta.
\end{abstract}

Termos para indexação: Bactris gasipaes, distúrbio nutricional, absorção de nutriente, nutriente mineral, tolerância ao sal.

\section{Mineral nutrition of peach palm seedlings under different salinity levels}

\begin{abstract}
The productive potential of plants may be affected by nutritional disorders induced by saline stress. The objective was to study the influence of different salinity levels on mineral nutrition of peach palm (Bactris gasipaes H.B.K.) seedlings in greenhouse conditions with nutritive solution. The experimental design was in randomized blocks, with seven treatments and four replications. The treatments consisted of the following dosages $\left(\mathrm{mmol} \mathrm{L}^{-1}\right): 0.0$ of Na and $0.5 \mathrm{of} \mathrm{Cl} ; 1.0$ of Na and 0.5 of $\mathrm{Cl}$ (control); and 5.0, 15.0, 30.0, 60.0 and 120.0 of NaCl. The content of the macronutrients varied with the salinity, there was reduction of $\mathrm{P}$ and $\mathrm{K}$ in the roots, $\mathrm{K}$ and $\mathrm{Ca}$ in the leaves, and $\mathrm{S}$ in different parts of the plants. Therefore, there was increase of $\mathrm{Na}$ and $\mathrm{Cl}$ in different parts of the plant. The enhancement of the ratios $\mathrm{Na} / \mathrm{K}, \mathrm{Na} / \mathrm{Ca}, \mathrm{Na} / \mathrm{Mg}, \mathrm{Cl} / \mathrm{N}, \mathrm{Cl} / \mathrm{P}$ and $\mathrm{Cl} / \mathrm{S}$ induced a nutritional imbalance in the plant.
\end{abstract}

Index terms: Bactris gasipaes, nutritional disturbance, nutrient absorption, mineral nutrients, salt tolerance.

\footnotetext{
(1) Aceito para publicação em 9 de julho de 2002.

Extraído da tese de doutorado apresentada pelo primeiro autor à Universidade Federal de Lavras (Ufla), Lavras, MG.

(2) Faculdade de Ciências Agrárias do Pará, Dep. de Ciência do Solo, Caixa Postal 917, CEP 66077-550 Belém, PA. E-mail: arfernan@fcap.br

(3)Ufla, Dep. de Ciência do Solo, Caixa Postal 37, CEP 37200-000 Lavras, MG. Bolsista do CNPq. E-mail: janicegc@ufla.br, niltcuri@ufla.br.

(4)Ufla, Dep. de Agricultura. E-mail: jeduardo@ufla.br

${ }^{(5)}$ Empresa de Pesquisa Agropecuária de Minas Gerais, Campus da Ufla. E-mail: paulotgg@ufla.br
}

\section{Introdução}

O Brasil é produtor e exportador de palmito em conserva, principalmente para os Estados Unidos e França. O Estado do Pará, através da exploração extrativista do açaizeiro (Euterpe oleracea Mart.), responde por 95\% do mercado nacional (Nogueira et al., 1995). A forma indiscriminada com que os açaizais passaram a ser explorados, em razão da grande demanda interna e externa do produto, submete esta espécie à ameaça de extinção. 
$\mathrm{Na}$ busca de alternativas para atender a um mercado crescente e que exige produtos de qualidade, oriundos de explorações racionais e ecológicas, surgiu a pupunheira (Bactris gasipaes H.B.K.), de ocorrência natural na Amazônia. Essa espécie apresenta elevado potencial de produção, tanto de palmito como de frutos com elevado valor nutritivo, mesmo em condições de baixa fertilidade natural dos solos. Destaca-se, ainda, por outras características agronômicas importantes, como precocidade, adaptabilidade a variações edafoclimáticas e capacidade de perfilhamento ao longo dos anos, além de produzir palmito de boa qualidade (Bovi, 1998).

Atualmente a pupunheira está sendo cultivada em diferentes regiões do Brasil, principalmente no $\mathrm{Su}$ deste. A expansão das áreas de cultivo, incluindo o semi-árido, tem sido acompanhada de uma melhoria do nível tecnológico, com a utilização de calagens, adubações e irrigações, o que tem possibilitado uma exploração mais precoce.

A intensificação do uso de fertilizantes e de irrigação tem sido a maior causa da salinização dos solos (Sonnoveld \& Welles, 1988), principalmente nas regiões áridas e semi-áridas. É um problema que cresce a cada ano em virtude da escassez de chuvas e da demanda sempre crescente de água e fertilizantes observada na agricultura moderna. Além disso, o aumento da população mundial tem forçado os produtores a utilizarem cada vez mais solos marginais, inclusive os salinos. Esses fatores têm aumentado a necessidade do desenvolvimento de tecnologias alternativas de uso desses solos, bem como da busca de espécies potencialmente promissoras para estas áreas.

Em ambientes salinos, o $\mathrm{NaCl}$ é o sal predominante e o que tem causado maior dano às plantas. Os efeitos sobre a nutrição mineral são decorrentes, principalmente, da toxicidade de íons, por causa da absorção excessiva de $\mathrm{Na}$ e $\mathrm{Cl}$, e do desequilíbrio nutricional causado pelos distúrbios na absorção ou distribuição dos nutrientes (Yahya, 1998). O grau de sensibilidade das plantas à salinidade é controlado pela absorção, translocação e exclusão dos íons $\mathrm{Na}$ e Cl. As plantas tolerantes absorvem esses íons rapidamente e os distribuem de forma uniforme nas diferentes partes da planta (Greenway \& Muns, 1980).
As espécies da família Arecaceae têm apresentado características favoráveis, pois o seu crescimento tem sido estimulado e sua produção aumentada pela presença do Na (Magat et al., 1993), e quantidades elevadas de $\mathrm{Cl}$ têm sido requeridas para expressar todo o seu potencial produtivo (Marschner, 1995; Sobral \& Leal, 1999). No entanto, não se conhecem as concentrações adequadas de $\mathrm{Cl}$ e de $\mathrm{Na}$ que possam estimular o crescimento e promover maior equilíbrio nutricional na pupunheira, como também se desconhece sua tolerância à salinidade.

Este trabalho teve como objetivo estudar a influência de diferentes níveis de salinidade, em solução nutritiva, sobre a nutrição mineral de mudas de pupunheira.

\section{Material e Métodos}

O experimento foi realizado em casa de vegetação do Departamento de Ciência do Solo na Universidade Federal de Lavras, Lavras, MG, situada a $918 \mathrm{~m}$ de altitude, $21^{\circ} 14^{\prime} \mathrm{S}$ de latitude e $45^{\circ} 0^{\prime} \mathrm{W}$ de longitude.

Os tratamentos constaram das seguintes doses (mmol L-1): 0,0 de $\mathrm{Na}$ e 0,5 de $\mathrm{Cl} ; 1,0$ de $\mathrm{Na}$ e $0,5 \mathrm{de} \mathrm{Cl}$ (controle); e 5,0, 15,0, 30,0, 60,0 e 120,0 de NaCl, correspondendo aos seguintes valores de condutividade elétrica (CE): 1,$28 ; 1,34 ; 2,06 ; 2,88 ; 4,42 ; 7,51$ e $13,20 \mathrm{dS} \mathrm{m}^{-1}$, respectivamente. O tratamento controle foi definido a partir da solução nutritiva determinada por Dufour et al. (1978) para o dendezeiro.

Adotou-se o delineamento em blocos casualizados, com sete tratamentos e quatro repetições. As unidades experimentais foram constituídas por um vaso contendo uma planta, com uma solução nutritiva básica de macronutrientes, composta de: $\mathrm{NO}_{3}{ }^{-}, 8 ; \mathrm{NH}_{4}{ }^{+}, 2 ; \mathrm{P}, 1 ; \mathrm{K}^{+}$, 2; $\mathrm{Ca}^{+2}, 2 ; \mathrm{Mg}^{+2}, 1,5 ; \mathrm{S}, 1 ; \mathrm{Na}^{+}, 1,0$ e Cl- $, 0,5$ mmol $_{\mathrm{c}} \mathrm{L}^{-1}$. As fontes dos nutrientes foram: $\mathrm{Ca}\left(\mathrm{NO}_{3}\right)_{2}, \mathrm{NH}_{4} \mathrm{NO}_{3}, \mathrm{NaNO}_{3}$, $\mathrm{Mg}\left(\mathrm{NO}_{3}\right)_{2}, \mathrm{KH}_{2} \mathrm{PO}_{4}, \mathrm{~K}_{2} \mathrm{SO}_{4}$ e $\mathrm{MgCl}_{2}$. As concentrações ( $\mathrm{m} \mathrm{L} \mathrm{L}^{-1}$ ) de micronutrientes e suas respectivas fontes foram: B, 0,20 $\left(\mathrm{H}_{3} \mathrm{BO}_{4}\right) ; \mathrm{Cu}, 0,05\left(\mathrm{CuSO}_{4} .5 \mathrm{H}_{2} \mathrm{O}\right) ; \mathrm{Fe}, 3,00$ (FeEDTA); $\mathrm{Mn}, \quad 0,35 \quad\left(\mathrm{MnSO}_{4} \cdot \mathrm{H}_{2} \mathrm{O}\right) ; \mathrm{Mo}, 0,02$ $\left(\left(\mathrm{NH}_{4}\right)_{6} \mathrm{Mo}_{7} \mathrm{O}_{27} .4 \mathrm{H}_{2} \mathrm{O}\right)$ e $\mathrm{Zn}, 0,05\left(\mathrm{ZnSO}_{4} \cdot 7 \mathrm{H}_{2} \mathrm{O}\right)$.

As plântulas com 60 dias depois de germinadas foram colocadas em bandejas coletivas com solução nutritiva, a $1 / 4$ da força iônica, durante 30 dias, e a $1 / 2$ da força iônica durante mais 30 dias. Após esse período, as plantas foram selecionadas e transferidas para vasos com $3 \mathrm{~L}$ de solução com força iônica total. Depois de 30 dias em tais recipientes, a solução foi modificada conforme os tratamentos, 
assim permanecendo por quatro meses. As soluções foram renovadas a cada 15 dias, nos dois primeiros meses e semanalmente, a partir do terceiro mês. Após este período, foram transferidas para vasos com capacidade para $9 \mathrm{~L}$, assim permanecendo por mais dois meses, perfazendo um período experimental de seis meses. Nesses recipientes, as soluções foram renovadas a cada 20 dias no primeiro mês e, a partir de então, a cada 15 dias.

A solução nutritiva foi mantida sob aeração constante durante todo o período experimental, assim como o seu volume foi mantido constante pela reposição diária de água deionizada, enquanto o $\mathrm{pH}$ da solução de cultivo foi monitorado e, quando atingia valores menores que cinco, a solução nutritiva dos vasos era trocada.

Depois de colhido, o material vegetal foi separado em raiz, estipe e folha. Após, todo o material vegetal foi lavado em água destilada corrente e secado em estufa com circulação de ar à temperatura de $65 \mathrm{a} 70^{\circ} \mathrm{C}$, até peso constante. A matéria seca das partes da planta foi pesada, moída e armazenada em frascos de vidro para as determinações químicas.

No extrato obtido por digestão nitroperclórica do material vegetal, determinaram-se os teores dos macronutrientes, do $\mathrm{Na}$ e do $\mathrm{Cl}$, conforme método descrito por Malavolta et al. (1997).

As relações do $\mathrm{Na}$ e do $\mathrm{Cl}$ com os nutrientes foram calculadas através dos teores nas diferentes partes da planta.

Os resultados foram submetidos à análise de variância, e como ocorreram diferenças significativas pelo teste $\mathrm{F}$ $(\mathrm{P}<0,05)$, estes foram submetidos a análises de regressão utilizando o sistema de análises estatísticas SANEST (Zonta \& Machado, 1991). Testaram-se diversos modelos para as equações, sendo a escolha baseada no coeficiente de determinação e na sua significância.

\section{Resultados e Discussão}

\section{Efeitos do $\mathrm{NaCl}$ nos teores de nutrientes nas folhas, estipes e raízes}

As doses de $\mathrm{NaCl}$ afetaram significativamente os teores dos nutrientes nas folhas, estipes e raízes das mudas de pupunheira, à exceção do $\mathrm{N}$ nas raízes, do $P$ nas folhas e nas estipes, e do Ca nas estipes (Tabela 1). As maiores alterações ocorreram em relação ao $\mathrm{P}$ e K nas raízes, $\mathrm{K}, \mathrm{Ca}$ e $\mathrm{Mg}$ nas folhas e $\mathrm{S}, \mathrm{Na}$ e $\mathrm{Cl}$ nas diferentes partes da planta.

Os teores de $\mathrm{N}$ nas folhas e nos estipes aumentaram nas doses de 15 a $60 \mathrm{mmol} \mathrm{L}^{-1}$ de $\mathrm{NaCl}$, em relação ao tratamento com $1,0 \mathrm{mmol} \mathrm{L}^{-1}$ de Na e $0,5 \mathrm{mmol}$ $\mathrm{L}^{-1}$ de $\mathrm{Cl}$ (controle) (Tabela 1). Estes resultados são similares aos de Saur et al. (1995), com Pinus pinaster Ait. de diferentes regiões da França. Já Al-Harbi (1995) constatou um aumento nos teores de $\mathrm{N}$ nas raízes até um nível de salinidade de $4,0 \mathrm{dS} \mathrm{m}^{-1}$, enquanto na parte aérea, os teores de $\mathrm{N}$ não foram afetados. Outros trabalhos têm demonstrado redução dos teores de $\mathrm{N}$ com o estresse salino $(\mathrm{Hu} \&$ Schmidhalter, 1997).

No entanto, o efeito da salinidade sobre o teor de $\mathrm{N}$ parece depender do nível de salinidade, do perío-

Tabela 1. Equação de regressão e coeficientes de determinação dos teores de nutrientes e de Na nas diversas partes de mudas de pupunheira, de acordo com as doses de $\mathrm{NaCl}$.

\begin{tabular}{clll}
\hline Nutriente & \multicolumn{1}{c}{ Folha } & \multicolumn{1}{c}{ Estipe } & \multicolumn{1}{c}{ Raiz } \\
\hline $\mathrm{N}$ & $\hat{\mathrm{Y}}=30,468+0,1583 \mathrm{X}-0,0015 \mathrm{X}^{2}$ & $\hat{\mathrm{Y}}=16,71+0,1785 \mathrm{X}-0,001 \mathrm{X}^{2}$ & $\hat{\mathrm{Y}}=20,665+0,0161 \mathrm{X}-0,0002 \mathrm{X}^{2}$ \\
& $\mathrm{R}^{2}=0,54^{* *}$ & $\mathrm{R}^{2}=0,77 *$ & $\mathrm{R}^{2}=0,04$ \\
$\mathrm{P}$ & $\hat{\mathrm{Y}}=3,826-0,007 \mathrm{X}+0,00007 \mathrm{X}^{2}$ & $\hat{\mathrm{Y}}=5,7928+0,0357 \mathrm{X}-0,0003 \mathrm{X}^{2}$ & $\hat{\mathrm{Y}}=6,3362-0,0909 \mathrm{X}+0,0004 \mathrm{X}^{2}$ \\
& $\mathrm{R}^{2}=0,03$ & $\mathrm{R}^{2}=0,22$ & $\mathrm{R}^{2}=0,97 * *$ \\
$\mathrm{~K}$ & $\hat{\mathrm{Y}}=13,2332-0,1108 \mathrm{X}+0,00088 \mathrm{X}^{2}$ & $\hat{\mathrm{Y}}=21,3702+0,13978 \mathrm{X}-0,00091 \mathrm{X}^{2}$ & $\hat{\mathrm{Y}}=17,599-0,0704 \mathrm{X}$ \\
& $\mathrm{R}^{2}=0,41^{* *}$ & $\mathrm{R}^{2}=0,59^{*}$ & $\mathrm{R}^{2}=0,88^{* *}$ \\
$\mathrm{Ca}$ & $\hat{\mathrm{Y}}=5,6555-0,0274 \mathrm{X}+0,0002 \mathrm{X}^{2}$ & $\hat{\mathrm{Y}}=4,7644-0,0129 \mathrm{X}+0,0001 \mathrm{X}^{2}$ & $\hat{\mathrm{Y}}=4,1758-0,011 \mathrm{X}+0,0001 \mathrm{X}^{2}$ \\
& $\mathrm{R}^{2}=0,36^{*}$ & $\mathrm{R}^{2}=0,30$ & $\mathrm{R}^{2}=0,47 *$ \\
$\mathrm{Mg}$ & $\hat{\mathrm{Y}}=1,9068-0,0163 \mathrm{X}+0,00013 \mathrm{X}^{2}$ & $\hat{\mathrm{Y}}=1,9369+0,0037 \mathrm{X}$ & $\hat{\mathrm{Y}}=1,4534-0,0018 \mathrm{X}+0,00005 \mathrm{X}^{2}$ \\
& $\mathrm{R}^{2}=0,40^{*}$ & $\mathrm{R}^{2}=0,36^{* *}$ & $\mathrm{R}^{2}=0,59^{*}$ \\
$\mathrm{~S}$ & $\hat{\mathrm{Y}}=2,6132-0,0259 \mathrm{X}+0,0002 \mathrm{X}^{2}$ & $\hat{\mathrm{Y}}=1,6933-0,0141 \mathrm{X}+0,00009 \mathrm{X}^{2}$ & $\hat{\mathrm{Y}}=4,0534-0,0399 \mathrm{X}+0,0003 \mathrm{X}^{2}$ \\
& $\mathrm{R}^{2}=0,45^{* *}$ & $\mathrm{R}^{2}=0,75^{* *}$ & $\mathrm{R}^{2}=0,89^{*}$ \\
$\mathrm{Na}$ & $\hat{\mathrm{Y}}=1,2538+0,2542 \mathrm{X}-0,0009 \mathrm{X}^{2}$ & $\hat{\mathrm{Y}}=1,1543+0,1338 \mathrm{X}$ & $\hat{\mathrm{Y}}=1,98+0,1592 \mathrm{X}-0,0005 \mathrm{X}^{2}$ \\
& $\mathrm{R}^{2}=0,97^{*}$ & $\mathrm{R}^{2}=0,99 * *$ & $\mathrm{R}^{2}=0,99^{* *}$ \\
$\mathrm{Cl}$ & $\hat{\mathrm{Y}}=5,7742+0,5297 \mathrm{X}-0,0016 \mathrm{X}^{2}$ & $\hat{\mathrm{Y}}=7,6585+0,765 \mathrm{X}-0,0032 \mathrm{X}^{2}$ & $\hat{\mathrm{Y}}=5,809+0,4467 \mathrm{X}-0,0025 \mathrm{X}^{2}$ \\
& $\mathrm{R}^{2}=0,94^{* *}$ & $\mathrm{R}^{2}=0,95^{* *}$ & $\mathrm{R}^{2}=0,83 * *$ \\
\hline
\end{tabular}

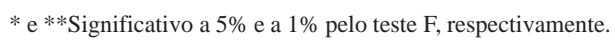


do em que a planta é submetida ao estresse salino e, principalmente, dos diferentes graus de tolerância das espécies ou genótipos (Al-Harbi, 1995).

Com o $\mathrm{P}$ ocorreu uma redução do teor nas raízes a partir de $15 \mathrm{mmol} \mathrm{L}^{-1}$ de $\mathrm{NaCl}$, o que está de acordo com os resultados obtidos por Aljuburi (1996), em palmáceas e Saur et al. (1995), em Pinus (Tabela 1). Tal fato pode estar relacionado a uma baixa atividade do $\mathrm{P}_{2} \mathrm{O}_{5}$ na solução, com o aumento do $\mathrm{NaCl}$ (Al-Karaki, 1997), que eleva a força iônica, ou a uma desordem nutricional induzida por elevados teores de $\mathrm{Cl}$ nos tecidos da planta, inibindo a absorção de P (Mor \& Manchanda, 1992, citados por Curtin et al., 1993).

Por sua vez, no tratamento controle, verificou-se menor teor de $\mathrm{P}$, nas diferentes partes da planta. A partir de $5 \mathrm{mmol} \mathrm{L}^{-1}$ de $\mathrm{NaCl}$ constatou-se maior absorção de fósforo. Possivelmente, maior quantidade foi exigida pela planta para a realização de suas funções metabólicas. Algumas pesquisas têm demonstrado que o estresse salino pode aumentar a necessidade de $\mathrm{P}$ de algumas culturas. Tal fato pode estar relacionado ao seu desempenho no armazenamento de energia e no transporte e particionamento de carboidratos (Gibson, 1988).

No caso do K, o aumento da concentração de $\mathrm{Na}$ na solução foi acompanhado por um declínio nos teores de $\mathrm{K}$ nas raízes e folhas, retratando menor absorção de $\mathrm{K}$ como conseqüência do aumento do estresse salino (Tabela 1). Tal redução é resultado do efeito antagônico do Na sobre o K, sugerindo uma competição entre esses íons pelos sítios de absorção na plasmalema (Marschner, 1995) ou um aumento do efluxo de $\mathrm{K}$ das raízes no meio de crescimento, por causa de distúrbios na integridade das membranas (Rengel, 1992).

A manutenção de níveis adequados de Ké essencial para a sobrevivência de plantas em ambientes salinos. O K é o mais proeminente soluto inorgânico da planta e como tal tem uma contribuição fundamental para o baixo potencial osmótico das células das raízes, o que é um pré-requisito para a pressão de turgor que determina o transporte de soluto via xilema e o balanço de água na planta (Marschner, 1995).

Os teores de $\mathrm{Ca}$ e de $\mathrm{Mg}$ nas folhas foram reduzidos, porém não atingiram proporções maiores com o aumento das doses de $\mathrm{NaCl}$ (Tabela 1). Elevadas concentrações de Na no meio externo podem reduzir a atividade do $\mathrm{Ca}$ na solução, resultando num decréscimo da quantidade de Ca disponível para a absorção pelas plantas (Cachorro et al., 1994). Já o decréscimo do teor de $\mathrm{Mg}$ pode estar relacionado a uma competição iônica com o Na (Hu \& Schmidhalter, 1997).

A redução significativa no teor de Ca nas folhas pode ter contribuído para um maior distúrbio nutricional na planta provocado pelos elevados teores dos íons tóxicos $\mathrm{Na}$ e $\mathrm{Cl}$, em virtude de maior porosidade das membranas induzida pelo $\mathrm{Na}$ (Rengel, 1992). Dentro deste contexto, pode-se inferir que o Ca tem um importante papel na resposta das plantas em condições salinas, já que o mesmo é essencial para manter a seletividade e a integridade das membranas, o que pode ser justificado pelos resultados de pesquisas indicando que a suplementação de $\mathrm{Ca}$, em tais condições, melhora o crescimento de várias espécies cultivadas (Cachorro et al., 1994).

Nas raízes, a elevação nas doses de $\mathrm{NaCl}$ promoveu um aumento dos teores de $\mathrm{Ca}$ em relação ao tratamento controle, o que pode ser decorrência do incremento do $\mathrm{Ca}$ estrutural necessário à manutenção da integridade da membrana plasmática em células de raízes desestruturadas pelo excesso de $\mathrm{Na}$ (Marschner, 1995).

Os teores de $\mathrm{S}$ foram reduzidos significativamente nas diferentes partes da planta, enquanto o $\mathrm{Na}$ e o $\mathrm{Cl}$ aumentaram (Tabela 1). Tal comportamento pode ser justificado por uma competição iônica entre o $\mathrm{Cl}$ e o S, resultando numa menor absorção e, ou, transporte para a parte aérea (Curtin et al., 1993). $\mathrm{O}$ aumento do teor de $\mathrm{Na}$ nas folhas foi maior que nos estipes e raízes, indicando a inexistência de mecanismos de exclusão ou de alocação de $\mathrm{Na}$, limitando o transporte para as folhas, características de espécies não tolerantes à salinidade (Yahya, 1998).

Os teores internos de $\mathrm{Cl}$ foram bem superiores aos do Na, dada a sua característica de ânion livre na planta, o que lhe confere mobilidade e transporte elevados (Marschner, 1995) (Tabela 1). Os maiores teores ocorreram nos estipes, seguidos de folhas e raízes. Estes resultados, tanto para $\mathrm{Na}$ como para $\mathrm{Cl}$, estão de acordo com os obtidos por Aljuburi (1996) com palmáceas.

Efeitos do $\mathrm{NaCl}$ nas relações $\mathrm{Na} / \mathrm{K}, \mathrm{Na} / \mathrm{Ca}, \mathrm{Na} /$ $\mathrm{Mg}, \mathrm{Cl} / \mathrm{N}, \mathrm{Cl} / \mathrm{P}$ e $\mathrm{Cl} / \mathrm{S}$ nas folhas, estipes e raízes

Com o aumento da salinidade, as relações do $\mathrm{Na}$ e do $\mathrm{Cl}$ com os outros nutrientes aumentaram significativamente, nas diferentes partes da planta (Figura 1). A manutenção de baixas relações 

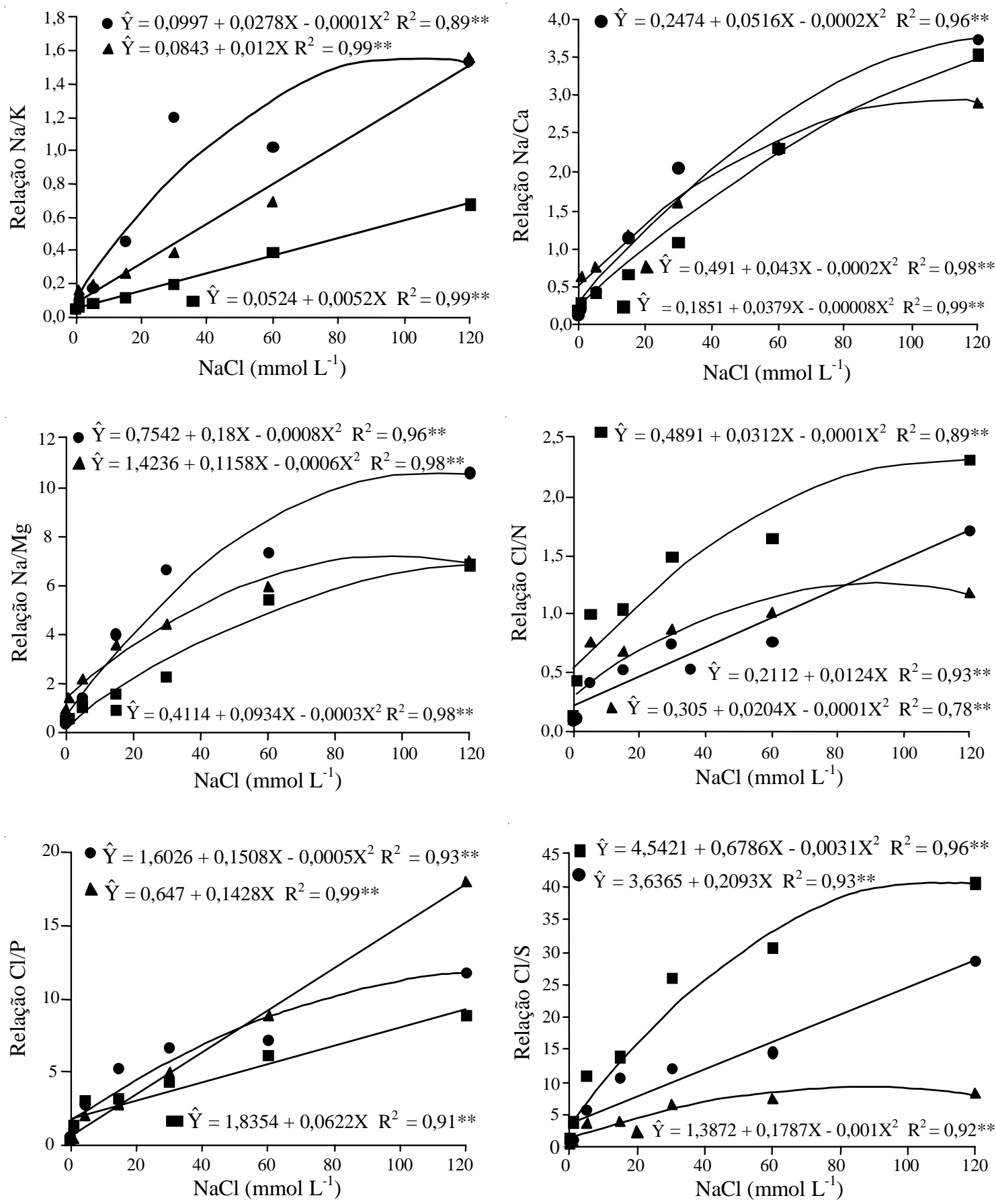

Figura 1. Relações $\mathrm{Na} / \mathrm{K}, \mathrm{Na} / \mathrm{Ca}, \mathrm{Na} / \mathrm{Mg}, \mathrm{Cl} / \mathrm{N}, \mathrm{Cl} / \mathrm{P}$ e Cl/S nas folhas $(\boldsymbol{O})$, estipes $(\boldsymbol{\square})$ e raízes $(\boldsymbol{\Delta})$ das mudas de pupunheira, em razão das doses de $\mathrm{NaCl}$. **Significativo a $1 \%$ pelo teste $\mathrm{F}$. 
$\mathrm{Na} / \mathrm{K}, \mathrm{Na} / \mathrm{Ca}$ e $\mathrm{Na} / \mathrm{Mg}$ é considerada por alguns autores como um importante critério na caracterização da tolerância das plantas à salinidade (Saur et al., 1995).

Relações $\mathrm{Na} / \mathrm{K}$ adequadas nos tecidos são necessárias para o funcionamento normal das células sob condições salinas (Greenway \& Muns, 1980), enquanto que uma relação $\mathrm{Na} / \mathrm{Ca}$ elevada na solução externa faz com que o $\mathrm{Na}$ desloque o $\mathrm{Ca}$ da membrana celular, induzindo a um aumento da permeabilidade e, por conseguinte, do teor intracelular de Na (Marschner, 1995). Neste sentido, Ashraf \& O'Leary (1997) relacionaram o maior crescimento de uma linhagem de girassol tolerante em relação a uma linhagem sensível à salinidade, à elevada relação $\mathrm{Ca} /$ $\mathrm{Na}$ nas folhas.

No tratamento controle, a relação $\mathrm{Na} / \mathrm{K}$ foi 0,101 , 0,064 e 0,158; a relação $\mathrm{Na} / \mathrm{Ca}$ foi 0,201, 0,285 e 0,652; e a relação $\mathrm{Na} / \mathrm{Mg}$ foi $0,548,0,567$ e 1,419 nas folhas, estipes e raízes, respectivamente.

A relação $\mathrm{Cl} / \mathrm{N}$ nas partes da planta pode constituir um importante indicativo da tolerância das mesmas à salinidade por sais de $\mathrm{Cl}$, já que são causados distúrbios quando este nutriente alcança teores internos excessivos, associados a teores internos de nitrato inadequados, o que é resultado de um efeito antagônico entre tais íons (Greenway \& Muns, 1980). Tal hipótese encontra suporte em trabalhos de Curtin et al. (1993), que constataram redução do teor de $\mathrm{Ne}$ aumento do teor de $\mathrm{Cl}$ em plantas de cevada (sensível à salinidade), enquanto em Kochia scoparia L. (tolerante) ocorreu um aumento do $\mathrm{N}$ e do $\mathrm{Cl}$, porém, deste último, pouco expressivo quando comparado ao ocorrido na espécie sensível; e de Kafkafi et al. (1992), em que cultivares de tomate e de melão tolerantes à salinidade apresentaram maior taxa de influxo de nitrato do que as sensíveis.

$\mathrm{O}$ aumento linear e bastante expressivo da relação $\mathrm{Cl} / \mathrm{P}$ nas raízes foi devido a um menor teor de $\mathrm{P}$ nas mesmas, resultado de menor absorção, seguida de uma continuada translocação, em razão de uma maior necessidade de P (Awad et al., 1990) nas folhas para realização de processos metabólicos, ou de um maior consumo energético da planta na tentativa de realizar um ajuste osmótico (Marschner, 1995).

Ao contrário da relação $\mathrm{Cl} / \mathrm{P}$, a menor relação
$\mathrm{Cl} / \mathrm{S}$ na planta ocorreu nas raízes, em conseqüência do maior teor de $\mathrm{S}$ nesta parte, o que sugere uma inibição do transporte deste nutriente pelo cloro. Neste sentido, Mor \& Manchanda (1992), citados por Grattan \& Grieve (1999), constataram que a salinidade provocada pelo $\mathrm{Cl}$ reduziu o conteúdo de $\mathrm{S}$ na palha de ervilha, enquanto nas raízes tal conteúdo aumentou.

No tratamento controle, a relação $\mathrm{Cl} / \mathrm{N}$ foi 0,103 , 0,423 e 0,133; a relação Cl/P foi 1,088, 1,379 e 0,453; e a relação $\mathrm{Cl} / \mathrm{S}$ foi $1,202,3,823$ e 0,637 nas folhas, estipes e raízes, respectivamente.

\section{Conclusões}

1. Quanto maior o nível de salinidade, menores são os teores de $\mathrm{P}$ e $\mathrm{K}$ nas raízes, os teores de $\mathrm{K} \mathrm{e} \mathrm{Ca}$ nas folhas, e o teor de $\mathrm{S}$ nas diferentes partes das plantas, e maiores os teores de $\mathrm{Na}$ e $\mathrm{Cl}$ nas diferentes partes das plantas de pupunheira.

2. As relações $\mathrm{Na} / \mathrm{K}, \mathrm{Na} / \mathrm{Ca}, \mathrm{Na} / \mathrm{Mg}, \mathrm{Cl} / \mathrm{N}, \mathrm{Cl} / \mathrm{Pe}$ $\mathrm{Cl} / \mathrm{S}$ aumentam com o aumento da salinidade e causam um desbalanço nutricional na planta, caracterizando a sensibilidade da pupunheira à salinidade.

\section{Referências}

AL-HARBI, A. R. Growth and nutrient composition of tomato and cucumber seedlings as affected by sodium chloride salinity and supplemental calcium. Journal of Plant Nutrition, New York, v. 18, n. 7, p. 1403-1416, 1995.

ALJUBURI, H. J. Effects of salinity and gibberellic acid on mineral concentration of date palm seedlings. Fruits, Paris, v. 51, n. 6, p. 429-435, Nov./Dec. 1996.

AL-KARAKI, G. N. Barley response to salt stress at varied levels of phosphorus. Journal of Plant Nutrition, New York, v. 20, n. 11, p. 1635-1643, 1997.

ASHRAF, M.; O'LEARY, J. W. Responses of a salttolerant and a salt-sensitive line of sunflower to varying sodium/calcium ratios in saline sand culture. Journal of Plant Nutrition, New York, v. 20, n. 2/3, p. 361-377, 1997.

AWAD, A. S.; EDWARDS, D. G.; CAMPBELL, L. C. Phosphorus enhancement of salt tolerance of tomato. Crop Science, Madison, v. 30, n. 1, p. 123-128, Jan./Feb. 1990.

BOVI, M. L. A. Palmito pupunha: informações básicas para cultivo. Campinas: Instituto Agronômico, 1998.50 p. (Boletim técnico, 173). 
CACHORRO, P.; OTIZ, A.; CERDÁ, A. Implications of calcium nutrition on the response of Phaseolus vulgaris L. to salinity. Plant and Soil, Dordrecht, v. 159, n. 2 , p. 205-212, Jan. 1994.

CURTIN, D.; STEPPUHN, H.; SELLES, F. Plant responses to sulfate and chloride salinity: growth and ionic relations. Soil Science Society of America Journal, Madison, v. 57, n. 5, p. 1304-1310, Sept./Oct. 1993.

DUFOUR, F.; QUENCEZ, P.; SCHMITY, G. Technique de culture en solutions nutritives du palmier à huile et du cocotier. Oléagineux, Paris, v. 33, n. 10, p. 485-490, oct. 1978.

GIBSON, T. S. Carbohydrate metabolism and phosphorus/ salinity interactions in wheat (Triticum aestivum L.). Plant and Soil, Dordrecht, v. 111, p. 25-35, 1988.

GRATTAN, S. R.; GRIEVE, C. M. Salinity-mineral relations in horticultural crops. Scientia Horticulturae, Amsterdam, v. 78, p. 127-157, 1999.

GREENWAY, H.; MUNS, R. Mechanisms of salt tolerance in crop plants. Plant Physiology, Rockville, v. 31, p. 149190, 1980.

HU, Y.; SCHMIDHALTER, U. Interactive effects of salinity and macronutrient level on wheat - part II: composition. Journal of Plant Nutrition, New York, v. 20, n. 9, p. 1169-1182, 1997.

KAFKAFI, U.; SIDDIQI, M. Y.; RITCHIE, R. J.; GLASS, A. D. M.; RUTH, T. J. Reduction of nitrate $\left({ }^{13} \mathrm{NO}_{3}\right)$ influx and nitrogen $\left({ }^{13} \mathrm{~N}\right)$ translocation by tomato and melon varieties after short exposure to calcium and potassium chloride salts. Journal of Plant Nutrition, New York, v. 15, p. 959-975, 1992.

MAGAT, S. S.; PADRONES, G. D.; ALFORJA, L. M. Residual effects of three chloride fertilizers on yield and leaf nutrient levels of coconuts grown on an inland soil of Davao (Mindanao, Philippines). Oleagineux, Paris, v. 48, p. 237-242, 1993.
MALAVOLTA, E.; VITTI, G. C.; OLIVEIRA, S. A. Avaliação do estado nutricional das plantas: princípios e aplicações. 2. ed. rev. atual. Piracicaba: Potafos, 1997. $319 \mathrm{p}$.

MARSCHNER, H. Mineral nutrition of higher plant. 2. ed. New York: Academy, 1995. 889 p.

NOGUEIRA, O. L.; CARVALHO, C. J. R.; MULLER, C. R.; GALVÃO, E. U. P.; SILVA, H. M.; RODRIGUES, J. E. L. F.; OLIVEIRA, M. P.; CARVALHO, J. E. U. de; ROCHA NETO, O. G. da; NASCIMENTO, W. M. O. do; CALZAVARA, B. B. A cultura do açaí. Brasília: Embrapa-SPI, 1995. 50 p. (Coleção Plantar, 26).

RENGEL, Z. Role of calcium in salt toxicity. Plant, Cell and Environment, Oxford, v. 15, p. 625-632, 1992.

SAUR, E.; LAMBROT, C.; LOUSTAU, D.; ROTIVAL, N.; TRICHET, P. Growth and uptake of mineral elements in response to sodium chloride of three provenances of maritime pine. Journal of Plant Nutrition, New York, v. 18 , n. 2, p. 243-256, 1995.

SOBRAL, L. F.; LEAL, M. L. S. Resposta do coqueiro à adubação com uréia, superfosfato simples e cloreto de potássio em dois solos do Nordeste do Brasil. Revista Brasileira de Ciência do Solo, Campinas, v. 23, p. 8589, 1999.

SONNOVELD, C.; WELLES, G. W. H. Yield and quality of rockwool-grown tomatoes as affected by variations in EC-value and climatic conditions. Plant and Soil, Dordrecht, v. 111, p. 37-42, 1988.

YAHYA, A. Salinity effects on growth and on uptake and distribution of sodium and some essential mineral nutrients in sesame. Journal of Plant Nutrition, New York, v. 21, n. 7, p. 1439-1451, 1998.

ZONTA, E. P.; MACHADO, A. A. Sistema de análise estatística para microcomputadores (SANEST). Pelotas: UFPel, 1991. 101 p. 